\title{
Processing pronominal pointing signs in German Sign Language: Neurophysiological evidence for the first mention effect
}

Anne Wienholz ${ }^{\mathrm{a}^{*}}$, Derya Nuhbalaoglu ${ }^{\mathrm{b}}$, Nivedita Manic ${ }^{\mathrm{c}}$, Annika Herrmann ${ }^{\mathrm{a}}$, Edgar Onea ${ }^{\mathrm{d}}$, Markus Steinbach

${ }^{\text {a }}$ Institute of German Sign Language and Communication of the Deaf, University of Hamburg, GorchFock-Wall 7, 20354 Hamburg, Germany

${ }^{\mathrm{b}}$ Department of Linguistics, Goethe University Frankfurt, Norbert-Wollheim-Platz 1, 60323 Frankfurt am Main, Germany

${ }^{\mathrm{c}}$ Psychology of Language Research Group, Georg Elias Müller Institute of Psychology, University of Goettingen, Goßlerstraße 14, 37073 Göttingen, Germany

${ }^{\mathrm{d}}$ Department of German Studies, University of Graz, Mozartgasse 8, 8010 Graz, Austria

${ }^{\text {e }}$ Department of German Philology, University of Goettingen, Käte-Hamburger-Weg 3, 37073 Göttingen, Germany

* Corresponding author at: Institute of German Sign Language and Communication of the Deaf, University of Hamburg, Gorch-Fock-Wall 7, 20354 Hamburg, Germany E-mail address: awien.research@gmail.com 


\begin{abstract}
Anaphoric pronoun resolution in spoken languages typically follows the first mention bias, according to which (personal) pronouns are interpreted as co-referring to the referents mentioned first in the discourse, typically introduced by the subject of the previous sentence. While the first-mention bias has been well investigated in spoken language processing, less is known about the presence of mechanisms underlying a similar bias in sign language processing. In sign languages, pronominal pointing signs (INDEX signs) are directed towards referential locations in the horizontal plane of the signing space typically associated with referents of the previous context. In German Sign Language (DGS), signers follow a ipsi-contralateral default pattern (right-left for right-handed signers) while assigning and tracking referents such that the first referent is overtly/covertly associated with the ipsilateral (right) and the second referent assigned to the contralateral (left) area of the signing space (Steinbach \& Onea, 2016; Wienholz et al., 2018). Hence, directing a pronoun to one or the other side can refer either to the first or second referent. The present event-related potential study examined the presence of a first mention effect during pronoun resolution in ambiguous contexts in DGS. Therefore, we presented participants with sentence sets containing two referents without overt localization in the first sentences and a pronominal sign at the beginning of the second sentence directed to either the ipsilateral (right) or contralateral (left) side of the signing space. Results show an N400 for contralateral INDEX signs compared to ipsilateral INDEX signs suggesting increased processing costs associated with the second referent being assigned to the contralateral space. Thus, the current study provides evidence for a first mention effect in German Sign Language and highlights its modality independent nature.
\end{abstract}


Keywords: German Sign Language (Deutsche Gebaerdensprache: DGS), event-related potentials, referential processing, first mention effect, N400 


\section{Introduction}

To process and comprehend an anaphoric pronoun, individuals are required to establish a co-reference relation between the pronoun and its corresponding antecedent. One way to establish co-referential relations during the anaphora resolution process in spoken languages is to rely on grammatical markers such as gender (Arnold et al., 2000). In unambiguous cases, the antecedent can be identified easily and the co-reference relation is resolved. Cross-linguistically, a number of different factors guide pronoun resolution in ambiguous contexts, including the so-called first mention effect, according to which ambiguous pronouns are interpreted as co-referential with the referent mentioned first in the discourse (Gernsbacher et al., 1989; Von Eckardt \& Potter, 1985).

Unlike spoken languages, sign languages make use of the linguistic potential of the three-dimensional signing space to guide referential relations. Referents are assigned to specific areas in the signing space. Directing a pronoun to that area in the subsequent discourse establishes a co-referential relation between the pronoun and the corresponding referent assigned to this area. A few studies investigating pronoun resolution preceded by overtly localized referents examine a first mention effect in sign languages as well (Emmorey \& Lillo-Martin, 1995; Krebs et al., 2018, 2019). However, in sign languages, referents do not have to be overtly localized in space. This may lead to supposedly ambiguous situations resulting in miscommunication, but this is not the case and communication happens successfully. One explanation might be the first mention bias applied by the addressee to resolve ambiguous contexts. Against this background, the current study investigates whether there is a first mention effect in German Sign Language in contexts where antecedents have not been overtly localized in the signing space. We briefly review previous literature related to the first mention effect in spoken languages and give a brief overview on pronoun resolution strategies in sign languages before presenting the current study. 


\section{First mention effects in spoken languages}

Previous experimental studies have observed the first mention effect across numerous languages. The first set of studies reporting this effect focused on English sentence pairs across a variety of different tasks. Using a probe recognition task, (Gernsbacher \& Hargreaves, 1988) examine the impact of linguistic factors, i.e., theta roles, sentence-initial position and syntactic subjecthood, on pronoun resolution. The data reveal a first mention effect reflected in faster reaction times to probes referring to the first mentioned referent than to probes referring to the second mentioned referent over and above the other linguistic factors investigated. Similarly, in a sentence completion task, (Crawley \& Stevenson, 1990) present participants with sentence fragments that either end with a pronoun or with a conjunction. In both comprehension and production tasks, participants prefer the first mentioned referent as the antecedent of the referential expressions, i.e., pronouns, independent of the sentence fragment's presentation in isolation or in discourse context. This preference is stronger in comprehension than in production. Similar effects are observed by (Crawley et al., 1990) in a reading and an assignment task showing that pronouns in object position within the target sentence show a strong preference towards a preceding subject, i.e., the first mentioned referent, but only if pronouns were ambiguous and no gender cue was available. Extending this to Spanish, (Carreiras et al., 1995) use a probe recognition task to examine the first mention preference in Spanish sentences, which allow greater flexibility in word order relative to English sentences. In a series of experiments, they present data showing a first mention preference in Spanish, which is reflected in faster reaction times to first mentioned referents irrespective of their syntactic status and the animacy of the arguments.

Other studies describe a similar effect, the subject preference, which identifies the antecedent of an ambiguous pronoun as the grammatical subject of the previous sentence 
(Bornkessel-Schlesewsky \& Schlesewsky, 2009). In a grammaticality judgement task on Dutch declarative sentences, (Frazier \& d'Arcais, 1989) observe that participants produce less errors and respond faster to subject-initial trials compared to object-initial trials, which is explained as reflecting a subject preference. For German relative clauses, (Schriefers et al., 1995) show, in a self-paced reading task, that relative clauses with an ambiguous sentenceinitial relative pronoun are preferentially interpreted as subject relative clauses under neutral conditions, i.e., no biased disambiguating element preceding the relative pronoun, reflected in shorter reading times to interpretations in keeping with this preference.

For languages such as English, in which the grammatical subject is typically also the first mentioned referent, it is difficult to disentangle the first mention effect from the subject preference effect. (Järvikivi et al., 2005) aim to disentangle these phenomena based on order of mention and on grammatical role in the processing of ambiguous pronouns. For this purpose, they examine the processing of pronouns in Finnish, a language with free word order and a gender neutral third person singular pronoun. The results show that subject and first mention preference both come into play in the resolution of ambiguous pronouns although the subject preference results in a stronger effect than the first mention preference.

\section{Pronoun resolution strategies in sign languages}

Sign languages exploit the signing space located in front of the signers' head and upper part of the body, to express various phonological, morphosyntactic, semantic and pragmatic functions (Perniss, 2012). The signing space is divided into different planes, among which, the horizontal plane plays an important role in indicating role shift (Lillo-Martin, 2012), temporal reference and topographic relations (Emmorey, 2002), plurals (Pfau \& Steinbach, 2006), contrast (van der Kooij et al., 2006), agreement (Padden, 1990) and, crucial for the current study, the spatial localization of referents (Lillo-Martin \& Klima, 1990). 
In sign languages, pronouns are expressed using pointing signs that are typically produced with an extended index finger directed towards a specific area in the signing space. The pointing sign (INDEX sign) is used to express various grammatical functions, such as determiners, locative adverbials or personal pronouns (Cormier et al., 2013; Pfau, 2011). In their pronominal function, pointing signs can either refer deictically to the signer, the addressee and third person referents physically present in the actual discourse, or anaphorically to third person referents not physically present in the actual discourse (for a review, see (Cormier, 2012)). Referring to physically present third person referents is easily achieved by pointing to their actual location in the communicative situation. In this case, the relation between the pronoun and its antecedent is established via deictic reference. However, in case a third person referent is not physically present, this antecedent has to be located on the horizontal plane of the signing space to establish an anaphoric relation between the antecedent and the corresponding pronoun. Therefore, a referent is assigned to an arbitrary locus in the horizontal plane, a referential locus (henceforth, R-locus) (Liddell, 1990; LilloMartin \& Klima, 1990; Sandler \& Lillo-Martin, 2006). Subsequently, a pronominal (pointing) sign can be directed towards that locus to pick up the established referent (Lillo-Martin \& Klima, 1990; Sandler \& Lillo-Martin, 2006).

Assignment to an R-locus is typically accomplished by a combination of manual, e.g., pointing signs (INDEX sign) or person agreement markers among others (Liddell, 1990; Pfau \& Steinbach, 2013), and nonmanual strategies, i.e., body leans, head movements or eye gaze towards the respective R-locus (Lillo-Martin, 1986; Winston, 1996). Yet, these strategies can also be used separately. Establishing R-loci in the horizontal plane appears to follow a particular pattern in keeping with the order of introduction of the referents, i.e., first and second mentioned, and directions, i.e., ipsi- and contralateral. A typical overt establishment of referents for right-handed signers of German Sign Language (DGS) involves the assignment of the first mentioned referent to the right (ipsilateral) and the second referent to the left 
(contralateral) side of the signing space (Steinbach \& Onea, 2016). A similar pattern has been described for Catalan Sign Language (Barberà, 2012), Italian Sign Language (Geraci, 2014), American Sign Language (ASL) (Sandler \& Lillo-Martin, 2006; Schlenker, 2013) and Israeli Sign Language (Meir \& Sandler, 2008). Studies on British Sign Language (Sutton-Spence \& Woll, 1999) and Danish Sign Language (Engberg-Pedersen, 1993) only mention the use of contrasting referents when using ipsi- and contralateral sides of the signing space, but do not elaborate on a specific order using these locations.

However, in cases where referents are not assigned overtly to an R-locus, one strategy signers rely on is a default pattern of referent localization in anaphora resolution. This default appears to follow the same strategy described for overt localization: first mentioned referents are assigned to the right/ipsilateral area and second mentioned referents to the left/contralateral side of the horizontal plane. This assumption is supported by empirical evidence reported in(Wienholz et al., 2018). In their study, (Wienholz et al., 2018) present participants with short strings of discourse consisting of two sentences while recording their electroencephalogram (EEG). The first sentence introduces two referents without overtly localizing them in space and the second sentence always starts with a pronominal pointing sign either directed to the right/ipsilateral or left/contralateral side of the horizontal plane followed by a neutral sign and a sentence-final sign that is semantically biased towards one of the referents in the first sentence. The data show an N400 effect for conditions containing a semantic mismatch, i.e., when the pronoun picked up the referent localized on the right side in the signing space while the sentence final sign in the second sentence referred to the referent established by default on the left side in space or vice versa. Thus, signers implicitly apply the default pattern leading to the observed effect. The study, therefore, provides experimental evidence for the assumption that the observed pattern for overt localization is a default pattern that is also applied in cases of covert localization in DGS. 


\section{First mention effects and subject preference in sign languages}

In sign language research, both a first mention effect and a subject preference have been investigated in a few previous studies. ${ }^{1}$ (Emmorey \& Lillo-Martin, 1995) present participants with signed sentences of ASL containing two referents followed by a second sentence containing either an overt pronoun co-referential with one of the previously introduced referents, a null pronoun licensed by verb agreement or no anaphora (control condition). They then measure reaction times for all conditions to probe signs representing one of the referents introduced in the first sentence. Responses in both pronoun conditions are faster than in the control condition suggesting that overt and null pronouns both reactivate their antecedents. Importantly for the current study, they find that reaction times to subject and object probe signs do not differ in the null pronoun condition leading the authors to argue that there is no first mention bias for ASL pronouns. They suggest that the absence of such an effect might be due to the phonological properties of verb morphology leading to simultaneous identification of subject and object or that the effect may only apply to explicitly expressed noun phrases and not to pronouns in general. However, (Emmorey \& Lillo-Martin, 1995) could only claim the absence of this effect for the null pronoun condition. Their claims ought not to be generalized to conditions including overt pronouns, since they do not present analyses of reaction times to probe signs that are either the first or second mentioned referent in the overt pronoun condition. Only such an analysis has the potential to reveal whether there is a first mention effect for overt pronouns.

\footnotetext{
${ }^{1}$ Recent work using offline methods such as production tasks show a second mention effect in different sign languages (Frederiksen, 2019; Nuhbalaoglu, 2018). However, since the current study uses online methods, a direct comparison of the results of these studies is not appropriate and will not be made here.
} 
Krebs and colleagues investigate the neural correlates underlying the processing of word order variations in Austrian Sign Language (ÖGS) (Krebs et al., 2018, 2019). Here, participants are presented with signed sentences in SOV and OSV orders, containing two arguments overtly linked to R-loci in the signing space followed by either an agreement verb or a plain verb in combination with an overt agreement marker, which is used in ÖGS to express agreement overtly with plain verbs. (Krebs et al., 2018) report lower acceptability ratings; longer reaction times and different ERPs to OSV compared to SOV structures, that are interpreted as reflecting increased processing of the OSV structure. These data, therefore, provide the first empirical evidence for the presence of a subject preference in ÖGS, thereby demonstrating its modality independence. As a follow-up, (Krebs et al., 2019) examine whether the observed subject preference can be modulated or eliminated in the context of sign language specific topic constructions. Behavioral data do not show any difference between conditions and word orders. However, differences in the ERPs to OSV compared to SOV structures are again taken as indexing increased processing costs for OSV structures. Together, these studies provide neurophysiological evidence for the presence of a subject preference even in sign language specific constructions.

Overall, these studies present data on the first mention effect and the subject preference as modality-independent mechanisms in language comprehension. However, it remains unclear whether the first mention effect and the subject preference are distinct processes or whether they describe the same or at least similar phenomena for sign languages. In the syntactic analysis of sign languages, it is still an ongoing debate how to define and use grammatical categories such as subject and object. Some sign languages are argued to determine their word order based on grammatical relations in that subjects typically precede objects (Li \& Thompson, 1976; Lillo-Martin, 1986; Meir, 2002). In contrast, other sign languages are reported to base their word order not on grammatical relations but on discoursefunctional relations (for a review, see (Leeson \& Saeed, 2012)). Consequently, the existence 
of grammatical subjects and objects are controversially discussed for these languages, with some people arguing that the categories subject and object are not relevant (or even misleading) for the linguistic description of sign languages (Bouchard \& Dubuisson, 1995; Li \& Thompson, 1976; Lillo-Martin, 1986). Despite this discussion, grammatical subjects precede grammatical objects in basic word order of most sign languages, thus complicating the differentiation between first mention effects and subject preference. Therefore, we will use the term first mention in our study as this term does not depend on specific syntactic assumptions and provides a theory neutral approach. Distinguishing between first mention and subject preference is not in the scope of this paper and is left for future research.

\section{The current study}

As presented above, previous research suggests the first mention effect to be a universal and modality-independent mechanism in language comprehension. Importantly, the effect is reported for unambiguous contexts where referents have been localized in the signing space. Therefore, the question arises whether a similar first mention effect can be observed for sign languages when referents have not been overtly localized in the signing space. To examine the first mention effect in DGS, we investigate whether pronominal INDEX signs directed to either the ipsilateral or the contralateral side of the horizontal plane evoke different neurophysiological effects. In contrast to (Krebs et al., 2018, 2019), referents in the present study will not be overtly localized in space. We are re-assessing the data from (Wienholz et al., 2018) by timelocking the analysis to an earlier position in the stimulus material as explained below.

While recording their EEG, participants are presented with short strings of discourse consisting of two sentences (same material as used by (Wienholz et al., 2018)). In the first sentence, two referents are introduced (e.g., PRIEST and GRANDMA as in subsequent examples 
(1) and (2)), but not assigned overtly to an R-Locus by any of the manual or nonmanual strategies mentioned above. ${ }^{2}$ The second sentence always starts with a pronominal pointing sign, i.e., the INDEX sign, followed by two additional signs. ${ }^{3}$ As already mentioned above, participants appear to follow a default pattern in interpreting the antecedents of the pronouns in the sentences (Wienholz et al., 2018). Based on this default pattern, the referent introduced first is implicitly assigned to the ipsilateral side whereas the second referent is established at the contralateral side. Thus, in the ipsilateral condition, the INDEX sign is directed to the ipsilateral/right (INDEX 3 a) side in the signing space expecting the continuation to refer to the first mentioned referent (Example 1). Sentences in the contralateral condition contained an INDEX sign directed to the contralateral/left side in the signing space anticipating a continuation referring to the second mentioned referent. In addition, all utterances contain a sentence-final predicate in first sentences, that does not require an additional agreement marker.

(1) Ipsilateral condition:

a. PRIEST GRANDMA MEET. INDEX3a HAVE BEARD.

'A priest meets a grandma. He has a beard.'

\footnotetext{
${ }^{2}$ Notational conventions: signs are glossed in small caps. In the presented example sentences, INDEX is
} always used as a pronoun referring back to one of the two referents introduced in the first sentence. Subscript ' 3 ' refers to the region in the horizontal plane used for third party referents whereas ' $a$ ' (right/ipsilateral) and 'b' (left/contralateral) denote the lateral sides in the horizontal plane. Glosses in bold indicate the pronoun and its (potential) antecedent. Note that DGS is assumed to be an SOV language (Happ \& Vorköper, 2006).

${ }^{3}$ The additional signs are not relevant for the purpose of this paper since they did not have an influence on the effects measured on the preceding INDEX sign. See (Wienholz et al., 2018) for a more detailed description of these signs. 
b. GRANDMA PRIEST MEET. INDEX 3 a HAVE BEARD.

'A grandma meets a priest. She has a beard.'

(2) Contralateral condition:

a. PRIEST GRANDMA MEET. INDEX 3 b HAVE BEARD.

'A priest meets a grandma. She has a beard.'

b. GRANDMA PRIEST MEET. INDEX 3 b HAVE BEARD.

'A grandma meets a priest. He has a beard.'

We expect the preference for either interpretation to be reflected in the modulation of the N400 ERP component. This negative-going component peaks between 300 and $500 \mathrm{~ms}$ following stimulus onset over central and parietal regions across either the left or right hemisphere (for a review, see (Kutas \& Federmeier, 2000, 2011)). Traditionally, the N400 is interpreted as reflecting processes underlying meaning processing and is modulated by lexical-semantic factors (Lau et al., 2008). However, this component has also been shown to reflect a reanalysis of grammatical functions, such as a subject-object reanalysis (Haupt et al., 2008). The same ERP component has been observed in sign language processing as well. In ASL, sentences containing or ending with a semantic violation evoke an N400 effect, just as the same violations for spoken languages do (Capek et al., 2009; Kutas et al., 1987). Similarly, semantic violations show a centrally distributed N400 in DGS (Hänel-Faulhaber et al., 2014; Hosemann et al., 2013). (Hosemann et al., 2013) observe the N400 in the transition phase, i.e., the phase between two signs, and therefore before the onset of the critical sign. This suggests that detection of semantic anomalies is already triggered by information contained in the transition phase. This finding is further supported by (Krebs et al., 2018, 2019) who show that the processing of object-initial structures in ÖGS evoke a negativity 
effect before the start of the disambiguating element even in sign language specific constructions.

Thus, regarding our study, if signers have a preference for one of the referents to continue with in subsequent discourse, we expect increased deflections for conditions violating this preference. In keeping with the first mention effect, we predict an increased negativity for the contralateral condition compared to the ipsilateral condition reflecting increased processing costs when referring to the second mentioned referent. However, if we observe increased brain activity in the ipsilateral condition with higher processing costs for first mentioned referents, signers do not show a preference for the first but rather for the second mentioned referent. Finally, ERPs might not differ across conditions indicating that there is no preference for either referent.

\section{Methods}

\section{Participants}

In this study, 21 (12 female, 9 male) deaf native signers of DGS (age range: 20-51 years; mean: 33 years) from different regions of Germany participated as paid volunteers. Eighteen were congenitally deaf and three became deaf between the age of 16 and 36 months. All participants had deaf parents or DGS input before the age of three years, were all righthanded (checked in a handedness test), had at least high school education level and normal or corrected-to-normal vision. During data analysis, one participant was excluded due to excessive eye movement artifacts. The Ethics Committee of the Institute of Psychology at the University of Goettingen provided ethics approval for the experiment. All participants received information about the EEG technique and the procedure in DGS and German and gave written signed consent prior to the experiment. 


\section{Materials}

In total, 240 stimulus sentences were presented comprising 160 experimental stimuli with 80 stimulus sentence sets for each condition (a full list of the stimulus material is provided in Appendix A) and an additional set of 80 filler sentences. Experimental conditions (see example 1 and 2 above) differ with respect to the direction of the INDEX sign used in the sentences. Thus, the ipsilateral condition contains an INDEX sign directed to the right, i.e., ipsilateral, side whereas the contralateral condition presents an INDEX sign directed to the left, i.e., contralateral, side on the horizontal plane. Furthermore, within each condition, the first mentioned referent was varied so that each of the two referents occurred once as the first and once as the second mentioned referent across participants.

A stimulus sentence set is composed of two naturally signed sentences containing three signs each. The sentences show no causal relation. In the first sentence, two referents were introduced without either manual or nonmanual localization, followed by a transitive non-localizing verb adhering to the neutral SOV word order in DGS. Referent combinations were used maximally twice while each referent occurred maximally ten times. Only animate referents were used so that the referent order could be reversed without changing the syntactic and semantic well-formedness of the sentences so that either referent could take the position of the agent/subject. Due to potential increased processing costs involved in the processing of compound signs, i.e., signs consisting of at least two signs to form one meaning, and proper names, i.e., fingerspelled or conventional name signs, these were never used as referents. To exclude localization with the verb, only non-localizing transitive verbs were chosen. Therefore, agreement verbs requiring spatial anchoring of referents and some non-localizing, i.e., plain, verbs obligatorily requiring an agreement marker directed toward the region in space where the referent was introduced were excluded (Rathmann, 2003). Moreover, verbs 
needed to allow for the reversed order of referents without changing the semantics and the appropriateness of the sentences. Noun pair-verb combinations occurred only once while verbs itself were repeated maximally four times.

Each second sentence started with an INDEX sign followed by two additional signs. The INDEX varied in the direction towards a corresponding region in the horizontal plane. In all sentences, the INDEX took the function of a pronoun referring either to the ipsilateral or the contralateral side of the signing space. Effects are measured at the INDEX so that participants are yet to see the rest of the second sentence at this particular time. Thus, the material following the INDEX ought not to influence the INDEX's processing and is not of interest for the purpose of the current study. ${ }^{4}$

Given dialectal variations of DGS (Eichmann \& Rosenstock, 2014; Langer, 2012; Macht \& Steinbach, 2019), we verified whether participants understood the referent signs as intended. The comprehension task was conducted prior to the experiment and ought not to impact the ERP data since we did not analyze ERP waveforms to these signs. All referent signs were presented as isolated single signs with their corresponding meaning (German word) as a subtitle. Participants had to indicate with a button press whether they understood the sign with the presented meaning even if they would not use the sign themselves. Familiarity of each participant with all items and familiarity of each item to the participants was analyzed. Participants on average knew $97 \%$ of the items (range: $74-100 \%$ ) and items were familiar to participants on average $97 \%$ as well (range: $76-100 \%$ ). Thus, no participant and no item were excluded from the analysis based on the comprehension task.

\footnotetext{
${ }^{4}$ The material in the second sentence following the INDEX is constructed the way that the second
} sentence unambiguously refers back to one of the referents introduced in the first sentence. Thus, the example sentences (1b) and (2a) result in a semantic mismatch at the final sign of the second sentence. These data are reported in (Wienholz et al., 2018). 
The sentence sets were signed by two male right-handed deaf native signers and recorded using a Sony HDR-CX550VE camcorder (25 frames per second) from frontal view. Verbs were signed in the neutral space in front of the signer to exclude overt spatial localization. Moreover, we controlled for the usage of nonmanuals during video recordings so that the signers avoided eye gaze shifts, body movements, head nods and eyebrow raise as their role in the processing of sign languages is still under-investigated. However, the signers used lexical nonmanuals and mouthing since both markers help to disambiguate between signs in DGS (Ebbinghaus \& Heßmann, 2001). There was a natural prosodic break between the two sentences. Videos were then edited using Adobe Premiere Pro CS6 such that the signer remained motionless for $2000 \mathrm{~ms}$ at the beginning and $1500 \mathrm{~ms}$ at the end of each video and that videos had a size of $1280 \times 720$ pixels. Each sentence set was recorded separately so that no further splicing within the video was necessary. Videos had a duration of $8550 \mathrm{~ms}$ on average $(7000-9320 \mathrm{~ms})$ with no significant difference of duration across conditions (Ipsilateral condition: $8539 \mathrm{~ms}$; Contralateral condition: $8561 \mathrm{~ms}$ ).

Additionally, a set of six practice items, four of them similar to the experimental and two similar to the filler sentences, was constructed and used to familiarize participants with the procedure. All practice items were constructed and recorded in the same way as sentences in the main experiment. Sign combinations in the practice experimental stimuli were not used in the main experiment.

\section{Procedure}

The study was conducted at the Experimental Sign Language Lab at the University of Goettingen. To guarantee a DGS language environment, a native signer of DGS supported the experimenters during testing. The experiment was preceded by a comprehension task and 
followed by a posttest. ${ }^{5}$ Participants were seated in a top-lit cabin on a chair at a distance of approximately $1 \mathrm{~m}$ from a 24 -inch screen. Stimuli were presented using the experimental software Presentation 18.1 (Neurobehavioral Systems, Inc.). Participants watched a DGS instruction video explaining that they would see multiple videos with DGS sentences and would be asked after each video to judge the sentences. Additionally, they were instructed to reduce body movements and, if possible, to blink after the presentation of each video. The practice items were presented to familiarize participants with the trial structure (Figure 1) and could be repeated as often as required by participants. Each trial started with a black blank screen for $500 \mathrm{~ms}$ followed by a video presenting one sentence set in the center of the screen in the natural speed of signing. Following each video, a question mark appeared signaling participants to provide their judgement of the presented sentences. Participants were instructed to judge the sentences based on their "gut feeling". ${ }^{6}$ They had to indicate, via button press on a Microsoft Xbox 360 Wired Controller, whether the sentence was good, i.e., pressing the green 'thumbs up' button, or bad, i.e., pressing the red 'thumbs down' button. There was no time limit given for the response time.

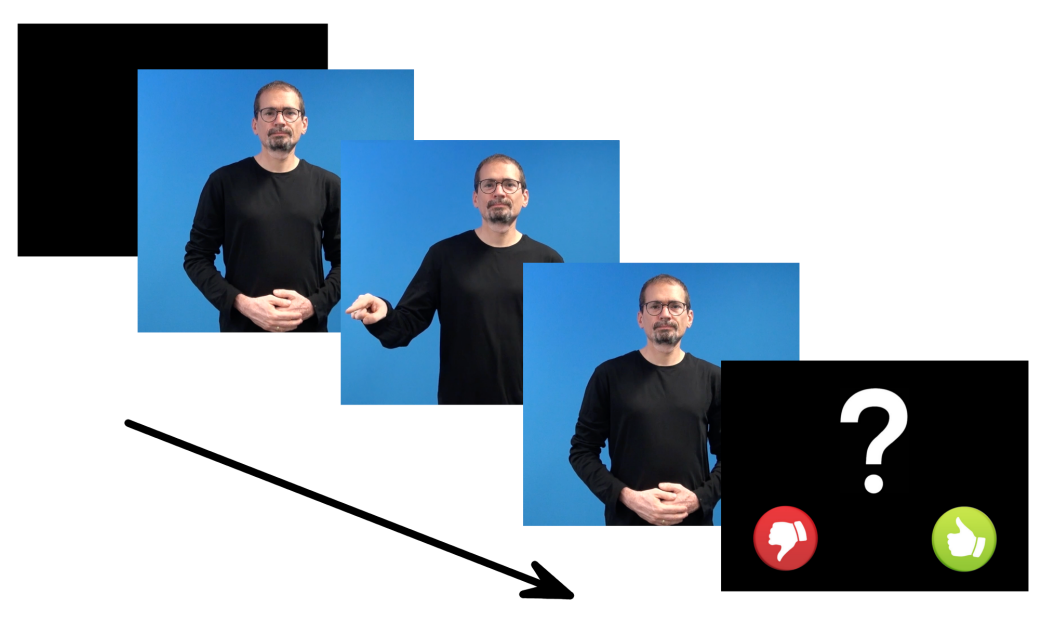

\footnotetext{
${ }^{5}$ For a description and the results of the posttest, see (Wienholz et al., 2018).

${ }^{6}$ Analysis and discussion of the behavioral data are presented in (Wienholz et al., 2018).
} 
Figure 1. Structure of a single trial in the main experiment

Stimuli sentence sets were pseudo-randomized into 10 blocks of 24 sets each in four different lists. Each block contained 16 experimental stimuli, i.e., eight from each condition, and eight filler sentences. Sentences within a block were all from different sentence sets and their order was randomized on-line by the experimental software.

\section{EEG-recording}

The EEG data were recorded using 64 active $\mathrm{Ag} / \mathrm{AgCl}$ electrodes placed according to the international 10-20 system and were amplified using an Active Two AD-Box (BioSemi B.V., Amsterdam, Netherlands) with a sampling rate of $2048 \mathrm{~Hz}$. The electrooculogram (EOG) was monitored using two external electrodes at the temple on each side (horizontal EOG) and one external electrode below the right eye in combination with electrode Fp2 on the cap (vertical EOG). Impedances were kept below $20 \mathrm{k} \Omega$ and data were re-referenced offline to linked mastoids. EEG data were analyzed using the ERPLAB toolbox plugin (LopezCalderon \& Luck, 2014) for MATLAB (The MathWorks, Inc.). First, the EEG signal was filtered using a $0.1-30 \mathrm{~Hz}$ band-pass filter and was down-sampled to $250 \mathrm{~Hz}$. Then, ERP averages were calculated for each participant for each electrode and condition. Two independent coders manually identified the following trigger positions for each video using the ELAN software. ${ }^{7}$ The coders mainly agreed on trigger positions. Cases of disagreement were discussed by the two coders until consensus was reached. No further inter-rater reliability was performed.

\footnotetext{
${ }^{7}$ The ELAN software is a linguistic annotation tool developed by the Max Planck Institute for
} Psycholinguistics, The Language Archive, Nijmegen, The Netherlands. http://tla.mpi.nl/tools/tla-tools/elan/ 
1. Sign offset: Last frame of the final hold of the sign preceding the critical sign before the hand gets relaxed again and before the movement of the transition phase starts. This trigger position was used for baseline correction.

2. Direction: The frame when the direction of the INDEX becomes clear due to the movement of the dominant hand towards one or the other side of the signing space. The sign might still point to one area, but the movement clearly indicates this sign's direction to another area.

ERPs were timelocked to the trigger 'direction' and baseline corrected $200 \mathrm{~ms}$ prior to sign offset triggers of the preceding sign as we aim to target the effects starting already at the introduction of the critical sign. We did not baseline correct to the time window immediately prior to the trigger 'direction', since processing of the sign already begins in the transition phase preceding the sign (Hosemann et al., 2013). Trials with artifacts, e.g., blinks or muscle movement, exceeding the artifact rejection threshold of $100 \mu \mathrm{V}$ were rejected before averaging. Based on previous sign language processing research reporting N400 effects and on results from t-tests, further analysis will focus on differences within the $400-500 \mathrm{~ms}$ time window (Hosemann et al., 2013; Krebs et al., 2018).

\section{Results}

For the analysis, lateral electrodes were combined according to the regions and hemisphere as follows: fronto-central (F3-FT7 \& F4-FT8), centro-temporal (C3-/TP7 \& C4TP8) and parietal-occipital (P3-PO7 \& P4-PO8) (see Appendix B for a schematic illustration of the regions). In the previously identified 400 - $500 \mathrm{~ms}$ time window, visual data inspection suggested a negativity for fronto-central and parietal-occipital regions on the right hemisphere 
(Figure 2). A $2 \times 3 \times 2$ ANOVA with the factors hemisphere (left, right), region (fronto-central, centro-temproal, parietal-occipital) and condition (ipsilateral, contralateral) revealed a significant main effect for region $(F(2,38)=9.178 ; p=.004)$ and a near significant interaction of hemisphere*condition $(F(1,19)=4.326 ; p=.051)$. The Greenhouse-Geisser correction was applied as required (Greenhouse \& Geisser, 1959).

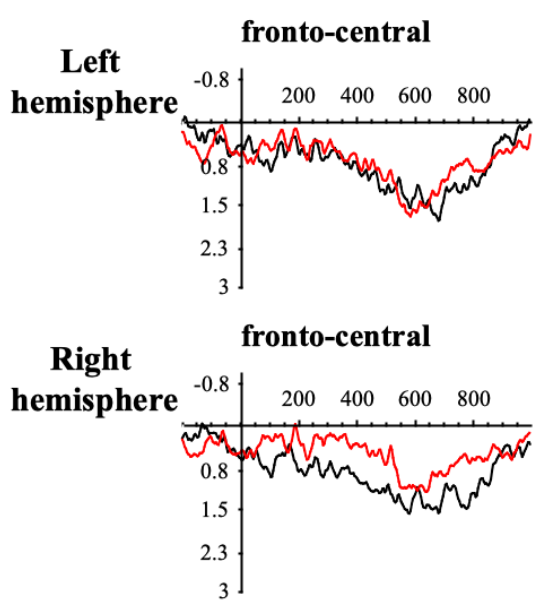

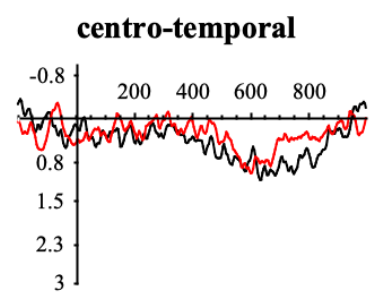

centro-temporal

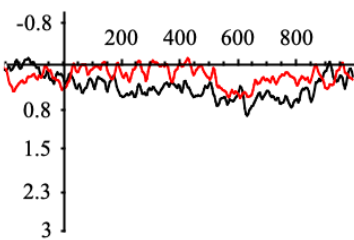

Ipsilateral condition

Contralateral condition

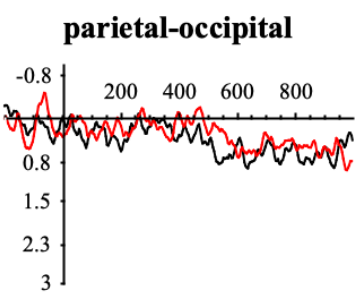

parietal-occipital

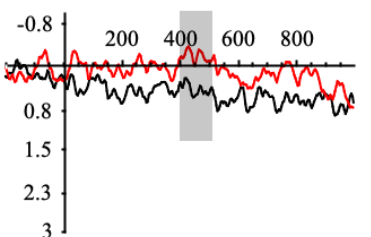

Figure 2. Grand average ERPs for ipsilateral (black) and contralateral (red) conditions timelocked to the trigger 'direction' for fronto-central, centro-parietal and parietal-occipital regions across left $(\mathrm{LH})$ and right hemisphere $(\mathrm{RH})$. Negativity is plotted upwards.

Splitting for hemisphere, a 3x2 ANOVA for the left and right hemisphere separately with the factors region (fronto-central, centro-temporal, parietal-occipital) and condition (ipsilateral, contralateral) revealed a significant main effect of region $(F(2,38)=6.924 ; p=$ .009) for the left hemisphere. For the right hemisphere, the analysis showed a significant main effect of region $(F(2,38)=7.822 ; p=.004)$ and a near-significant main effect of condition $(F(1,19)=3.717 ; p=.069)$. Paired samples t-tests comparing ipsilateral and contralateral conditions only revealed a significant difference on the right hemisphere for the parietal- 
occipital region $(t(19)=2.236 ; p=.038)$ with the contralateral condition $(M=-0.436)$ being more negative relative to the ipsilateral condition $(M=0.783)$, providing weak evidence for the suggestion of increased processing costs when the contralateral INDEX is presented, i.e., when the location consistent with the second mentioned referent is presented as the continuation of the discourse. No other comparisons reached significance.

\section{Discussion}

This study examined the presence of the first mention effect in DGS by investigating neurophysiological effects evoked by pronominal pointing signs. We presented participants with two-sentence discourses containing two referents. Both referents were introduced without any overt localization cue in the first sentence followed by an ipsilateral or contralateral pronominal pointing sign in the second sentence. Based on the default pattern for the assignment of referents to an R-locus in the signing space and depending on its direction (Wienholz et al., 2018), the pronoun refers either to the first or second referent. Thus, ipsilateral pronominal pointing signs refer to the first referent and contralateral pointing signs refer to the second mentioned referent. The results offer evidence of increased negative deflections timelocked to the trigger position 'direction' of the pronominal pointing sign in the $400-500 \mathrm{~ms}$ time window for the contralateral condition compared to the ipsilateral condition in the parietal-occipital region on the right hemisphere. In particular, we found increased processing costs associated with processing of the contralateral pointing sign, the location associated by default with the second mentioned referent. This could be interpreted as evidence for a first mention effect for DGS.

The interpretation of the observed N400 as a first mention effect in DGS is based on previous findings showing that DGS users follow a default pattern in assigning referents to Rloci covertly (Wienholz et al., 2018). According to this default (Steinbach \& Onea, 2016), the 
referent introduced first in the discourse is assigned to the ipsilateral side, while the referent mentioned second is assigned to the contralateral side of the signing space. Extending this to the stimuli presented in our study, the INDEX sign referring to the ipsilateral side picks up the first mentioned referent, while the INDEX sign referring to the contralateral side picks up the second mentioned referent. The N400 triggered by the contralateral INDEX in the current study suggests that participants expect the second sentence to continue with the first mentioned referent, i.e., the ipsilateral INDEX sign, and incur additional processing costs following violation of this expectation due to presentation of the contralateral INDEX sign.

As outlined above, (Krebs et al., 2018) argue for a subject preference in ÖGS, such that discourse continuations that pick up the object of a previous sentence cause additional processing costs relative to continuations that pick up the subject of this sentence. There are however, three crucial differences between the current study and (Krebs et al., 2018). First, we note that referents were not overtly localized in the current study. Furthermore, we investigated the influence of ambiguous pronoun usage across sentences, while (Krebs et al., 2018) examined word order variations. Finally, we ensured that we only used non-localizing verbs as these do not use localizations in the space to express referential relations in contrast to agreement verbs that do rely on spatial information. Despite these differences, the results of both studies hint towards a first mention effect in DGS. In particular, we note that in DGS, basic word order is described as SOV (Happ \& Vorköper, 2006). Our stimulus material, in keeping with this basic word order, similarly had the first referent taking the position of the grammatical subject and the second referent as grammatical object. To this extent, the effect in our study could equally be interpreted as an effect of subject preference, such that the pronoun in the ipsilateral condition would refer back to the grammatical subject while the contralateral pronoun would pick up the grammatical object. Therefore, the effect would reflect increased processing costs when the discourse continues with the object, indicating a subject preference in discourse continuations. This interpretation would be in line with 
observations reported by (Krebs et al., 2018) for ÖGS and comparable with the forced choice data from (Frederiksen \& Mayberry, 2017) reporting a subject preference for ASL. At present, we are unable to tease apart whether subject preference and first mention effects are two distinct processes or just different ways of referring to the same phenomenon especially in languages with SOV word order. Further research in sign languages with variable word order could be used to tell apart these two concepts.

A similar difference triggered by the processing of ipsilateral or contralateral INDEX signs was observed by (Wienholz et al., 2018) using the same stimulus material. Comparing (congruent) trials based on the direction of the INDEX sign revealed increased processing cost for the contralateral direction between 500-600 ms. One difference between the results in this study and the previous work is that the effect was observed only across the left hemisphere in (Wienholz et al., 2018). Given that the current study measured ERPs timelocked to when the direction of the sentence-initial INDEX sign was clearly predictable, compared to ERPs timelocked to the beginning of the sentence-final sign in (Wienholz et al., 2018), we suggest that the left hemisphere specific effect in (Wienholz et al., 2018) may have been triggered by processing of additional contextual information prior to sign processing.

Indeed, while both hemispheres of the brain are shown to be involved in language processing (for a review, see (Burgess \& Chiarello, 1996)), the right hemisphere plays an especially important role in discourse processing. Initial evidence in keeping with this suggestion comes from studies examining patients with right hemisphere brain damage, who display difficulties in discourse level processing. Difficulties range from impairment in topic maintenance and metaphor comprehension, while nevertheless showing full understanding of single sentences and single words (Burgess \& Chiarello, 1996; St George et al., 1999). Neurophysiological studies similarly provide evidence for right hemispheric involvement (in addition to left hemisphere activity) in processing semantic and pragmatic information, suggesting that this hemisphere is associated with comparing new information to previously 
obtained context information and maintaining a coherent discourse (Federmeier \& Kutas, 1999; St George et al., 1999). The few case studies on sign languages that are available suggest a similar involvement of the right hemisphere in discourse processing (Hickok et al., 1999). Data obtained from signers with right hemispheric brain damage show inconsistent use of R-loci, i.e., switching between horizontal and midsaggital plane, difficulties in topic maintenance and role shift while production and comprehension of signs and sentences continues to be unimpaired (Emmorey, 2002; Hickok et al., 1999; Loew et al., 1997). Taken together, the N400 effect observed here and its right hemispheric distribution across the scalp might be attributed to the discourse processing focus of the current study. Therefore, the neurophysiological data might indicate right hemispheric involvement in semantic-pragmatic discourse processing in sign languages.

One final caveat that needs to be addressed here is the possibility that the N400 effect reported here may be triggered purely by processing of the spatial location of the referential expression. In other words, it is possible that the observed ERP differences are due to differential processing of the ipsilateral or contralateral area in the signing space rather than due to the pronoun disambiguation as suggested here. We suggest that this explanation is unlikely, given that the two distinct areas in the space should evoke an effect of visuospatial processing, which typically occur quite early, usually within the first $200 \mathrm{~ms}$ post-stimulus (Luck, 2005). The absence of temporal overlap between the visual components expected in such a case and our findings suggests that the reported effect is unlikely to be due to early differences in visuospatial processing of right and left areas of the signing space. Equally, we note that a further alternative interpretation of the observed effect is an index of mismatch between the expected continuation and the presented stimulus expectation, specifically targeting the visual mismatch negativity (MMN, e.g., (Kimura et al., 2011)). However, we note that the visual MMN peaks earlier, between 250-400 ms post-stimulus, and consequently does not offer a reasonable interpretation of our observed effect, similar to other earlier visual 
components. To rule out perceptual effects (Neville et al., 1997), the experiment needs to be tested with non-signers watching the sign model pointing to either side of the signing space. If this group shows similar effects as the DGS signers, physical differences of the pointing signs rather than their linguistic information would yield the observed effect. In contrast, if they perform differently, this would suggest that signers associate the spatial directions with language-specific discourse-pragmatic function. We leave this for future research.

\section{Conclusion}

This study provides further evidence that the first mention effect is modalityindependent and applies to spoken and sign languages to the same extent. In particular, we show that participants incurred increased processing costs when the discourse continuations referred to the second mentioned referent in the discourse relative to the first mentioned referent. We note that while the phenomena of first mention effect and subject preference can be distinguished in some spoken languages, the extent to which the two effects can be separated within a specific sign language or whether the occurrence of the effect can be generalized across sign languages remains unclear. Since sign languages do not show overt case marking (Sandler \& Lillo-Martin, 2006), they might behave similar to their spoken language equivalents such as English. We rather need to test passive constructions where the object can be mentioned first or other constructions where objects are fronted to be able to disentangle the two phenomena. However, there is more work needed to look at the basic word order and its variations in DGS as well as discussing the terminology of grammatical subject and object in sign languages in general. Additionally, further studies should focus on examining effects evoked in other sign language specific structures such as topic constructions to allow for generalization of these findings. 


\section{Acknowledgments}

We would like to thank our colleagues and deaf informants for their help during material development and video recording, our student assistants for their support during experiment preparation and our deaf research assistant for support during testing and data acquisition. Most importantly, we are grateful to all the individuals who participated in this study.

This work was supported by the Deutsche Forschungsgemeinschaft (DFG, German Research Foundation; Priority Program (SPP 1727) XPrag.de: New Pragmatic Theories based on Experimental Evidence, research project Discourse Referents in Space - Anaphora Resolution in German Sign Language), the Leibniz ScienceCampus for Primate Cognition and the Deutsche Forschungsgemeinschaft (DFG, German Research Foundation) - Project number 254142454/GRK 2070.

\section{Declaration of interest statement}

No potential conflict of interest was reported by the authors.

\section{References}

Arnold, J. E., Eisenband, J. G., Brown-Schmidt, S., \& Trueswell, J. C. (2000). The rapid use of gender information: Evidence of the time course of pronoun resolution from eyetracking. Cognition, 76(1), B13-B26. https://doi.org/10.1016/s00100277(00)00073-1

Barberà, G. (2012). The Meaning of Space in Sign Language. Reference, Specificity and Structure in Catalan Sign Language Discourse. De Gruyter Mouton. 
Bornkessel-Schlesewsky, I., \& Schlesewsky, M. (2009). Processing syntax and morphology: A neurocognitive perspective (Vol. 6). Oxford University Press.

Bouchard, D., \& Dubuisson, C. (1995). Grammar, order \& position of wh-signs in Quebec Sign Language. Sign Language Studies, 87(1), 99-139.

Burgess, C., \& Chiarello, C. (1996). Neurocognitive mechanisms underlying metaphor comprehension and other figurative language. Metaphor and Symbol, 11(1), 67-84. https://doi.org/10.1207/s15327868ms1101_4

Capek, C. M., Grossi, G., Newman, A. J., McBurney, S. L., Corina, D. P., Röder, B., \& Neville, H. J. (2009). Brain systems mediating semantic and syntactic processing in deaf native signers: Biological invariance and modality specificity. Proceedings of the National Academy of Sciences, 106(21), 8784-8789. https://doi.org/10.1073/pnas.0809609106

Carreiras, M., Gernsbacher, M. A., \& Villa, V. (1995). The advantage of first mention in Spanish. Psychonomic Bulletin \& Review, 2(1), 124-129. https://doi.org/10.1037/e665412011-201

Cormier, K. (2012). Pronouns. In R. Pfau, M. Steinbach, \& B. Woll (Eds.), Sign Language: An International Handbook (pp. 227-244). De Gruyter Mouton.

Cormier, K., Schembri, A., \& Woll, B. (2013). Pronouns and pointing in sign languages. Lingua, 137, 230-247. https://doi.org/10.1016/j.lingua.2013.09.010

Crawley, R. A., \& Stevenson, R. J. (1990). Reference in single sentences and in texts. Journal of Psycholinguistic Research, 19(3), 191-210. https://doi.org/10.1007/BF01077416

Crawley, R. A., Stevenson, R. J., \& Kleinman, D. (1990). The use of heuristic strategies in the interpretation of pronouns. Journal of Psycholinguistic Research, 19(4), 245-264. https://doi.org/10.1007/bf01077259

Ebbinghaus, H., \& Heßmann, J. (2001). Sign language as multidimensional communication: Why manual signs, mouthings, and mouth gestures are three different things. In P. 
Boyes Braem \& R. Sutton-Spence (Eds.), The hands are the head of the mouth (pp. 133-151). Signum Verlag.

Eichmann, H., \& Rosenstock, R. (2014). Regional variation in German Sign Language: The role of schools (re-)visited. Sign Language Studies, 14(2), 175-202. https://doi.org/10.1353/sls.2014.0001

Emmorey, K. (2002). Language, Cognition, and the Brain: Insights From Sign Language Research. Lawrence Erlbaum and Associates.

Emmorey, K., \& Lillo-Martin, D. (1995). Processing spatial anaphora: Referent reactivation with overt and null pronouns in American Sign Language. Language and Cognitive Processes, 10(6), 631-653. https://doi.org/10.1080/01690969508407116

Engberg-Pedersen, E. (1993). Space in Danish Sign Language: The Semantics and Morphosyntax of the Use of Space in a Visual Language. Signum Verlag.

Federmeier, K. D., \& Kutas, M. (1999). Right words and left words: Electrophysiological evidence for hemispheric differences in meaning processing. Cognitive Brain Research, 8(3), 373-392. https://doi.org/10.1016/s0926-6410(99)00036-1

Frazier, L., \& d'Arcais, G. B. F. (1989). Filler driven parsing: A study of gap filling in Dutch. Journal of Memory and Language, 28(3), 331-344. https://doi.org/10.1016/0749$596 x(89) 90037-5$

Frederiksen, A. T. (2019). Referential Cohesion in American Sign Language: ModalitySpecific and Modality-General Influences [UC San Diego]. https://escholarship.org/uc/item/6rr7w3m7

Frederiksen, A. T., \& Mayberry, R. I. (2017, February 20). Pronominalizing Subjects: Referentially Empty Pronouns in ASL? Workshop on Anaphora Resolution on Sign and Spoken Languages - Theoretical and Experimental Dimensions, Göttingen, Germany.

Geraci, C. (2014). Spatial syntax in your hands. In J. Iyer \& L. Kusmer (Eds.), Proceedings of 
the Forty-Fourth Annual Meeting of the North East Linguistic Society (pp. 123-134). GLSA. https://sites.google.com/site/carlogeraci76/home

Gernsbacher, M. A., \& Hargreaves, D. J. (1988). Accessing sentence participants: The advantage of first mention. Journal of Memory and Language, 27(6), 699-717. https://doi.org/10.1016/0749-596X(88)90016-2

Gernsbacher, M. A., Hargreaves, D. J., \& Beeman, M. (1989). Building and accessing clausal representations: The advantage of first mention versus the advantage of clause recency. Journal of Memory and Language, 28(6), 735-755. https://doi.org/10.1016/0749-596x(89)90006-5

Greenhouse, S. W., \& Geisser, S. (1959). On methods in the analysis of profile data. Psychometrika, 24(2), 95-112. https://doi.org/10.1007/bf02289823

Hänel-Faulhaber, B., Skotara, N., Kügow, M., Salden, U., Bottari, D., \& Röder, B. (2014). ERP correlates of German Sign Language processing in deaf native signers. BMC Neuroscience, 15(1), 62. https://doi.org/10.1186/1471-2202-15-62

Happ, D., \& Vorköper, M.-O. (2006). Deutsche Gebärdensprache: Ein Lehr-und Arbeitsbuch. Fachhochschulverlag.

Haupt, F. S., Schlesewsky, M., Roehm, D., Friederici, A. D., \& Bornkessel-Schlesewsky, I. (2008). The status of subject-object reanalyses in the language comprehension architecture. Journal of Memory and Language, 59(1), 54-96. https://doi.org/10.1016/j.jml.2008.02.003

Hickok, G., Wilson, M., Clark, K., Klima, E. S., Kritchevsky, M., \& Bellugi, U. (1999). Discourse deficits following right hemisphere damage in deaf signers. Brain and Language, 66(2), 233-248. https://doi.org/10.1006/brln.1998.1995

Hosemann, J., Herrmann, A., Steinbach, M., Bornkessel-Schlesewsky, I., \& Schlesewsky, M. (2013). Lexical prediction via forward models: N400 evidence from German Sign Language. Neuropsychologia, 51(11), 2224-2237. 
https://doi.org/10.1016/j.neuropsychologia.2013.07.013

Järvikivi, J., van Gompel, R. P., Hyönä, J., \& Bertram, R. (2005). Ambiguous Pronoun Resolution: Contrasting the First-Mention and Subject-Preference Accounts. Psychological Science, 16(4), 260-264. https://doi.org/10.1111/j.09567976.2005.01525.x

Kimura, M., Schröger, E., \& Czigler, I. (2011). Visual mismatch negativity and its importance in visual cognitive sciences. Neuroreport, 22(14), 669-673. https://doi.org/10.1097/wnr.0b013e32834973ba

Krebs, J., Malaia, E., Wilbur, R. B., \& Roehm, D. (2018). Subject preference emerges as cross-modal strategy for linguistic processing. Brain Research, 1691, 105-117. https://doi.org/10.1016/j.brainres.2018.03.029

Krebs, J., Malaia, E., Wilbur, R. B., \& Roehm, D. (2019). Interaction between topic marking and subject preference strategy in sign language processing. Language, Cognition and Neuroscience, 1-19. https://doi.org/10.1080/23273798.2019.1667001

Kutas, M., \& Federmeier, K. D. (2000). Electrophysiology reveals semantic memory use in language comprehension. Trends in Cognitive Sciences, 4(12), 463-470. https://doi.org/10.1016/s1364-6613(00)01560-6

Kutas, M., \& Federmeier, K. D. (2011). Thirty years and counting: Finding meaning in the N400 component of the event-related brain potential (ERP). Annual Review of Psychology, 62, 621-647. https://doi.org/10.1146/annurev.psych.093008.131123

Kutas, M., Neville, H. J., \& Holcomb, P. J. (1987). A preliminary comparison of the N400 response to semantic anomalies during reading, listening and signing. Electroencephalography and Clinical Neurophysiology Supplement, 325-330.

Langer, G. (2012). A colorful first glance at data on regional variation extracted from the dgscorpus: With a focus on procedures. 5th Workshop on the Representation and Processing of Sign Languages: Interactions between Corpus and Lexicon, 101-108. 
https://www.sign-lang.uni-hamburg.de/dgskorpus/files/inhalt_pdf/LREC_2012_Colours.pdf

Lau, E. F., Phillips, C., \& Poeppel, D. (2008). A cortical network for semantics:

(De)constructing the N400. Nature Reviews Neuroscience, 9(12), 920-933. https://doi.org/10.1038/nrn2532

Leeson, L., \& Saeed, J. (2012). Word order. In R. Pfau, M. Steinbach, \& B. Woll (Eds.), Sign Language: An International Handbook (pp. 245-265). De Gruyter.

Li, C. N., \& Thompson, S. L. (1976). Subject and topic: A new typology of language. In C. N. Li (Ed.), Subject and topic (pp. 459-489). Academic Press.

Liddell, S. K. (1990). Four functions of a locus: Re-examining the structure of space in ASL. In C. Lucas (Ed.), Sign language research: Theoretical issues (pp. 176-198). Gallaudet University Press.

Lillo-Martin, D. (1986). Two kinds of null arguments in American Sign Language. Natural Language \& Linguistic Theory, 4(4), 415-444. https://doi.org/10.1007/bf00134469

Lillo-Martin, D. (2012). Utterance reports and constructed action in sign and spoken languages. In R. Pfau, M. Steinbach, \& B. Woll (Eds.), Sign Language: An International Handbook (pp. 365-387). De Gruyter Mouton.

Lillo-Martin, D., \& Klima, E. S. (1990). Pointing Out Differences: ASL Pronouns in Syntactic Theory. In S. D. Fischer \& P. Siple (Eds.), Theoretical Issues in Sign Language Research, Volume 1: Linguistics. University of Chicago Press.

Loew, R. C., Kegl, J. A., \& Poizner, H. (1997). Fractionation of the components of role play in a right-hemispheric lesioned signer. Aphasiology, 11(3), 263-281. https://doi.org/10.1080/02687039708248469

Luck, S. J. (2005). An introduction to the event-related brain potential technique. MIT Press. Macht, C., \& Steinbach, M. (2019). Regionalsprachliche Merkmale in der Deutschen Gebärdensprache. In J. Herrgen \& J. E. Schmidt (Eds.), Sprache und Raum. Ein 
internationales Handbuch der Sprachvariation (pp. 916-937). De Gruyter Mouton.

Meir, I. (2002). A cross-modality perspective on verb agreement. Natural Language \& Linguistic Theory, 20(2), 413-450. https://doi.org/10.1023/A:1015041113514

Meir, I., \& Sandler, W. (2008). A language in space: The story of Israeli Sign Language. Lawrence Erlbaum Associates.

Neville, H. J., Coffey, S. A., Lawson, D. S., Fischer, A., Emmorey, K., \& Bellugi, U. (1997). Neural Systems Mediating American Sign Language: Effects of Sensory Experience and Age of Acquisition. Brain and Language, 57(3), 285-308. https://doi.org/10.1006/brln.1997.1739

Nuhbalaoglu, D. (2018). Comprehension and production of referential expressions in German Sign Language: An empirical approach. [Doctoral dissertation, University of Goettingen]. https://ediss.uni-goettingen.de/handle/21.11130/00-1735-0000 -0003C152-6

Padden, C. (1990). The relation between space and grammar in ASL verb morphology. In C. Lucas (Ed.), Sign language research: Theoretical issues (pp. 118-132). Gallaudet University Press.

Perniss, P. (2012). Use of sign space. In R. Pfau, M. Steinbach, \& B. Woll (Eds.), Sign Language: An International Handbook (pp. 412-431). De Gruyter Mouton.

Pfau, R. (2011). A point well taken: On the typology and diachrony of pointing. In D. J. Napoli \& G. Mathur (Eds.), Deaf around the world: The impact of language (pp. 144163). Oxford University Press.

Pfau, R., \& Steinbach, M. (2006). Pluralization in sign and in speech: A cross-modal typological study. Linguistic Typology, 10(2), 135-182. https://doi.org/10.1515/LINGTY.2006.006,

Pfau, R., \& Steinbach, M. (2013). PERSON Climbing up a Tree (and other Adventures in Sign Language Grammaticalization). Sign Language \& Linguistics, 16(2), 189-220. 
https://doi.org/10.1075/s1l.16.2.04pfa

Rathmann, C. (2003). The optionality of agreement phrase: Evidence from German Sign Language (DGS). In W. E. Griffin (Ed.), The role of agreement in natural language: Proceedings of the Fifth Annual Texas Linguistics Society Conference (pp. 181-192). Texas Linguistic Forum.

Sandler, W., \& Lillo-Martin, D. (2006). Sign Language and Linguistic Universals. Cambridge University Press.

Schlenker, P. (2013). Anaphora: Insights from Sign Language. In S. R. Anderson, J. Moeschler, \& F. Reboul (Eds.), The Language-Cognition Interface: Actes du 10e Congrès International des Linguistes. Librairie Droz.

Schriefers, H., Friederici, A. D., \& Kuhn, K. (1995). The processing of locally ambiguous relative clauses in German. Journal of Memory and Language, 34(4), 499-520. https://doi.org/10.1006/jmla.1995.1023

St George, M., Kutas, M., Martinez, A., \& Sereno, M. I. (1999). Semantic integration in reading: Engagement of the right hemisphere during discourse processing. Brain, 122(7), 1317-1325. https://doi.org/10.1093/brain/122.7.1317

Steinbach, M., \& Onea, E. (2016). A DRT Analysis of Discourse Referents and Anaphora Resolution in Sign Language. Journal of Semantics, 33(3), 409-448. https://doi.org/10.1093/jos/ffv002

Sutton-Spence, R., \& Woll, B. (1999). The linguistics of British Sign Language: An Introduction. Cambridge University Press.

van der Kooij, E., Crasborn, O., \& Emmerik, W. (2006). Explaining prosodic body leans in Sign Language of the Netherlands: Pragmatics required. Journal of Pragmatics, 38(10), 1598-1614. https://doi.org/0.1016/j.pragma.2005.07.006

Von Eckardt, B., \& Potter, M. C. (1985). Clauses and the semantic representation of words. Memory \& Cognition, 13(4), 371-376. https://doi.org/10.3758/bf03202505 
Wienholz, A., Nuhbalaoglu, D., Mani, N., Herrmann, A., Onea, E., \& Steinbach, M. (2018). Pointing to the right side? An ERP study on anaphora resolution in German Sign Language. PLoS ONE, 13(9), e0204223. https://doi.org/10.1371/journal.pone.0204223

Winston, E. A. (1996). Spatial mapping in ASL discourse. In D. M. Jones (Ed.), Assessing Our Work: Assessing Our Worth (pp. 1-28). CIT Conference Proceedings. 


\section{APPENDIX B}

Schematic illustration of the regions of interest (ROIs): There are six regions in total with 3 regions per hemisphere consisting of six electrodes each. Regions are marked by same colors.

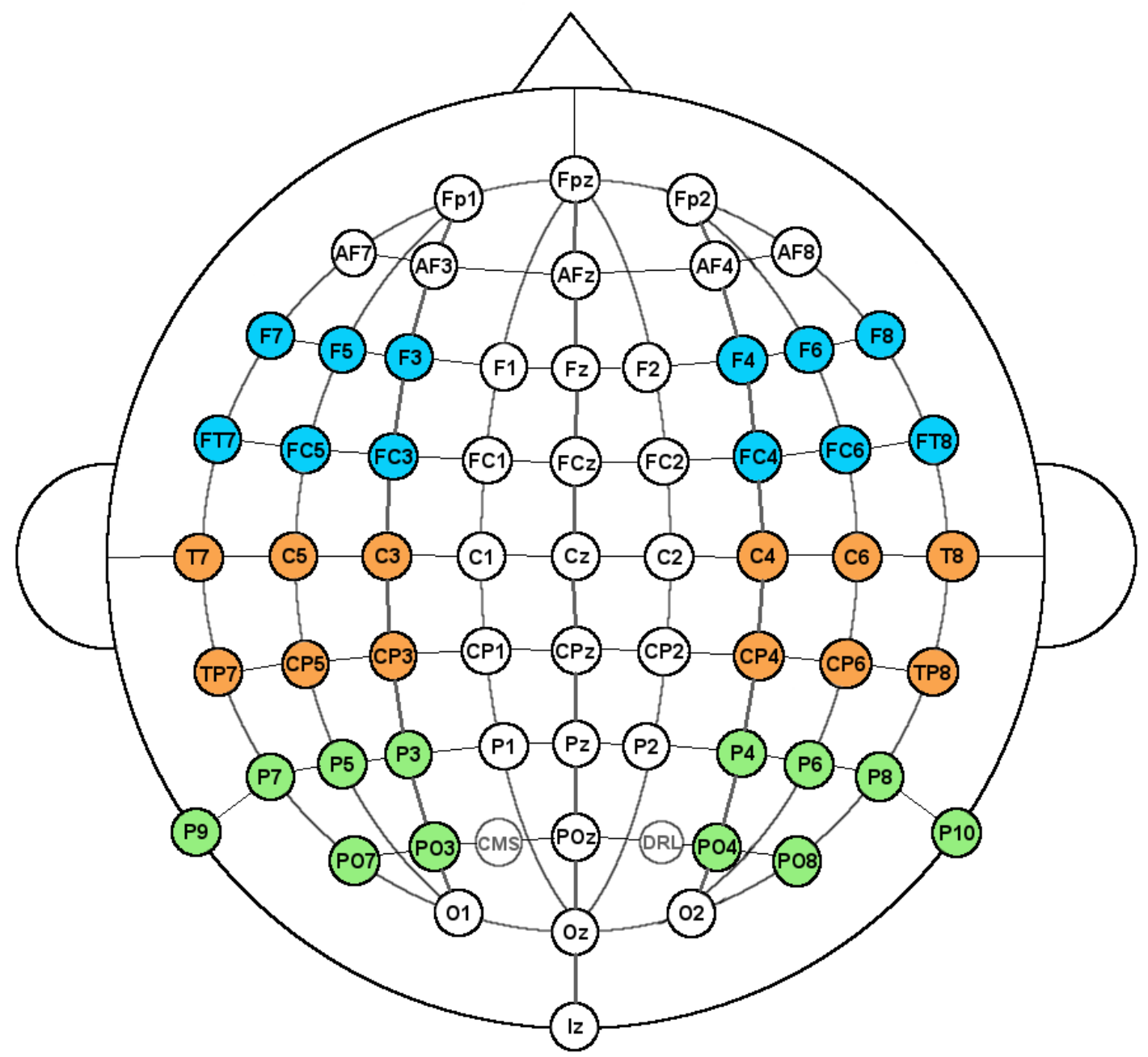

